\title{
Tafel zur Berechnung der Störungsfunction für die äussersten kleinen Planeten.
}

\section{Von $H$. Ludendorff.}

Bei der Entwicklung der Störungsfunction nach Herrn Gyldén's Methode treten gewisse Coefficienten $\beta_{n}^{(s)}$ auf, welche durch die Gleichung

$$
\beta_{n}^{(s)}=\int_{\pi}^{2} \int_{0}^{1 / 2 \pi} \frac{\sin ^{2 n} \varphi}{\left(1-\alpha^{2} \sin ^{2} \varphi\right)^{1 / 2} s} d \varphi
$$

definirt werden.

Hierbei ist $a=\frac{a}{a^{\prime}}$ das Verhältniss zweier Constan. ten, von denen die eine $(a)$ der mittleren Entfernung des gestörten, und die andere $\left(a^{\prime}\right)$ der mittleren Entfernung des störenden Körpers, also des Jupiter, entspricht. Es sind die von Herrn Gyldén ,Protometer genannten Grössen.

Um die directe, ziemlich mithsame Berechnung dieser Coefficienten zu ersparen, haben Herr Wellmann ") und, in noch ausführlicherer Weise, Herr Masal $\dagger$ ) Tafeln $\operatorname{der} \beta_{n}{ }^{(s)}$ berechnet. Beide Tafeln geben jedoch nicht die Möglichkeit, die $\beta_{n}^{(s)}$ auch für die äussersten kleinen Planeten, deren mittlere Bewegung kleiner als etwa 500" ist, insbesondere also für diejenigen vom Hilda-Typus, zu berechnen. Das Interesse, welches gerade diese Planeten in Folge der bei einigen von ihnen fast strengen Commensurabilitäl der mittleren Bewegung mit derjenigen Jupiters verdienen, liess es winschenswerth erscheinen, die erwähnte Lücke auszu- fullen, und ich habe daher auf Anregung von Herrn Brendel die nachstehende Tafel berechnet. Wegen der sehr grossen Störungen, welche diese Planeten erleiden, kann man nur schwer einen guten Näherungswerth der Constanten $\alpha$ erlangen, und man kann sich daher gezwungen sehen, nachträglich auf einen, von dem ursprünglich angenommenen abweichenden Werth von $\alpha$ überzugehen. Dieser Uebergang ist mit Hülfe der Tafel leicht $2 u$ bewerkstelligen.

Die Berechnung der Tafel geschah nach einem Verfahren, auf welches ich von Herrn Brendel aufmerksam gemacht wurde. Setzt man nämlich

$$
A_{n}^{(s)}=\left(1-\alpha^{2}\right)^{1 / 2 s} \beta_{n}^{(s)} \text {, }
$$

so ist

$$
\begin{aligned}
& A_{n}^{(s)}=\frac{2}{\pi} \int_{0}^{1 / 2 \pi} \frac{\sin ^{2 n} \varphi}{\left(1+2 \varepsilon \cos ^{2} \varphi\right)^{1 / 2} s} \mathrm{~d} \varphi, \\
& 2 \varepsilon=\frac{\alpha^{2}}{I-\alpha^{2}} .
\end{aligned}
$$

Durch die Absonderung des Factors $\left(1-\alpha^{2}\right)^{1 / 2} s$ wird, wie zuerst Herr Wellmann in seinem oben erwähnten Aufsatz erkannt hat, die Convergenz der Reihenentwicklung erheblich verstärkt.

Es ist ferner :

$$
\frac{\mathrm{d}^{m} A_{n}^{(s)}}{\mathrm{d} \varepsilon^{m}}=(-\mathrm{I})^{m} s \cdot(s+2)(s+4) \cdots(s+2 m-2) \frac{2}{\pi} \int_{0}^{1 / 9}\left(\frac{\cos ^{2} \varphi}{1+2 \varepsilon \cos ^{2} \varphi}\right)^{m} \frac{\sin ^{2 n} \varphi \mathrm{d} \varphi}{\left(1+2 \varepsilon \cos ^{2} \varphi\right)^{1 / 2} s} .
$$

Berechnet man nun für einen speciellen Werth $\alpha_{0}$ von $\alpha$, dem der Werth $\varepsilon_{0}$ von $\varepsilon$ entsprechen möge, die Grössen $A_{n}^{(1)}$ nebst ihren Ableitungen, und bezeichnet diese Werthe mit

$$
\left(A_{n}^{(s)}\right)_{0}, \cdots,\left(\frac{\mathrm{d}^{m} A_{n}^{(s)}}{\mathrm{d} \varepsilon^{m}}\right)_{\mathrm{o}} \cdots
$$

so kann man die $A_{n}^{(s)}$ für einen beliebigen Werth von $\alpha$ resp. $\varepsilon$ nach der Taylor'schen Reihe

$$
A_{n}^{(s)}=\left(A_{n}^{(s)}\right)_{0}+\frac{\mathrm{I}}{\mathrm{I} !}\left(\frac{\mathrm{d} A_{n}^{(s)}}{\mathrm{d} \varepsilon}\right)_{0} \Delta+\frac{\mathrm{I}}{2 !}\left(\frac{\mathrm{d}^{2} A_{n}^{(s)}}{\mathrm{d} \varepsilon^{2}}\right)_{0} \Delta^{2}+\cdots
$$

wo $\Delta=\varepsilon-\varepsilon_{0}$ ist, bestimmen.

Zur Berechnung der Tafel wurde $\log \alpha_{0}=9.8810000$ gewählt, ein Werth, welcher sehr nahe dem Planeten (153) Hilda entspricht; der zugehörige Werth von $\varepsilon$ ist $\varepsilon_{0}=$ 0.6851038 . Für diesen Werth wurden die in den Coeff. cienten der Reihe auftretenden bestimmten Integrale nach dem von Herrn Harzer in seinen, Untersuchungen über einen speciellen Fall des Problems der drei Körper» angegebenen, sehr zweckmässigen Verfahren berechnet. In der
Tafel finden sich nur die Reihen für $A_{n}{ }^{(1)}(n=0$ bis 12$)$, $A_{12}{ }^{(3)}, A_{12}{ }^{(5)}, A_{12}{ }^{(7)}$. Hat man. diese Grössen aus der Tafel entnommen, so ergeben sich die entsprechenden $\beta_{n}^{(s)}$ aus:

$$
\beta_{n}^{(s)}=\frac{\mathrm{I}}{\left(\mathrm{I}-\alpha^{2}\right)^{1 / 2 s}} A_{n}^{(s)}
$$

Die $\beta_{n}{ }^{(3)}, \beta_{n}{ }^{(3)}, \beta_{n}{ }^{(7)}$ fùr $n<12$ erhält man alsdann mit Hülfe der von Herrn Gyldén gegebenen Recursionsformel :

") Wellmann, Tafeln zur Berechnung der Störungsfunction der kleinen Planeten, A. N. Bd. I27, Nr. 3040.

t) Masal, Table de l'Intégrale $\int_{0}^{1 / 2 \pi} \frac{\sin ^{2 n} \varphi}{\left(1-\alpha^{2} \sin ^{2} \varphi\right)^{1 / 2} s} d \varphi$. (Astr. iakttagelser och undersökningar anstälda pa Stockholm's Obs., IV, 5). 


$$
\beta_{n}^{(s+2)}=\beta_{n}^{(s)}+\alpha^{2} \beta_{n+1}^{(s+2)}
$$

Da man im weiteren Verlauf der Rechnung nicht die $\beta_{n}{ }^{(s)}$ selber, sondern nur die mit denselben durch die Relation :

$$
\begin{aligned}
& \gamma_{n .0}=\frac{\alpha^{n+1}}{\left(1-\alpha^{2}\right)^{1 / 2}} A_{n}{ }^{(1)} \\
& \gamma_{10.2}=\frac{3}{8} \frac{a^{15}}{\left(1-\alpha^{2}\right)^{5 / 2}} A_{12}{ }^{(5)}
\end{aligned}
$$

und dann die andern $\gamma_{n . \sigma}$ nach der folgenden, leicht herzuleitenden Recursionsformel bestimmt:

$$
\gamma_{n . \sigma}=\frac{2 \sigma-1}{2 \sigma} \alpha \gamma_{n+1 . \sigma-1}+\alpha \gamma_{n+1 . \sigma} \text {. }
$$

Trotz dieser bequemen Recursionsformeln kann es gerade bei den Planeten vom Hilda-Typus nützlich sein,

$$
\gamma_{n, \sigma}=\frac{1 \cdot 3 \cdot 5 \cdots(2 \sigma-1)}{2 \cdot 4 \cdot 6 \ldots 2 \sigma} \alpha^{n+2 \sigma+1} \beta_{n+\sigma}^{(2 \sigma+1)}
$$

verbundenen Grössen braucht, so verfährt man vielleicht zweckmässiger, wenn man aus den tabulirten $A_{n}^{(s)}$ die zu. gehörigen $\gamma_{n . \sigma}$ berechnet, nämlich :

$$
\begin{aligned}
& \gamma_{11,1}=\frac{1}{2} \frac{\alpha^{14}}{\left(1-\alpha^{2}\right)^{3 / 2}} A_{12}{ }^{(3)} \\
& \gamma_{9.3}=\frac{5}{16} \frac{\alpha^{16}}{\left(1-\alpha^{2}\right)^{7 / 2}} A_{12}{ }^{(7)}
\end{aligned}
$$

auch die $A_{n}^{(s)}$ fur $s=3,5,7$ und $n<12$ zu tabuliren, um eine unter Umständen nothwendige Veränderung des Werthes von $\alpha$ möglichst leicht vornehmen zu können. Um eine solche Erweiterung der Tafel zu erleichtern, habe ich die folgenden Formeln hergeleitet, welche leicht verificirt werden können:

$$
\begin{aligned}
& A_{n}^{(s+2)}=\frac{1}{1+2 \varepsilon} A_{n}^{(s)}+\frac{2 \varepsilon}{1+2 \varepsilon} A_{n+1}^{(s+2)} \\
& \frac{\mathrm{d} A_{n}{ }^{(s+2)}}{\mathrm{d} \varepsilon}=\frac{s+2}{s} \frac{\mathrm{I}}{\mathrm{I}+2 \varepsilon} \frac{\mathrm{d} A_{n}{ }^{(s)}}{\mathrm{d} \varepsilon}+\frac{2 \varepsilon}{\mathrm{I}+2 \varepsilon}-\frac{\mathrm{d} A_{n+1}^{(s+2)}}{\mathrm{d} \varepsilon} \\
& \frac{\mathrm{d}^{2} A_{n}^{(s+2)}}{\mathrm{d} \varepsilon^{r}}=\frac{s+4}{s} \frac{\mathrm{r}}{\mathrm{I}+2 \varepsilon} \frac{\mathrm{d}^{2} A_{n}^{(s)}}{\mathrm{d} \varepsilon^{2}}+\frac{2 \varepsilon}{\mathrm{i}+2 \varepsilon}-\frac{\mathrm{d}^{2} A_{n+1}^{(s+2)}}{\mathrm{d} \varepsilon^{2}} \\
& \frac{\mathrm{d}^{m} A_{n}^{(s+2)}}{\mathrm{d} \varepsilon^{m}}=\frac{s+2 m}{s} \frac{1}{1+2 \varepsilon} \frac{\mathrm{d}^{m} A_{n}^{(s)}}{\mathrm{d} \varepsilon^{m}}+\frac{2 \varepsilon}{1+2 \varepsilon} \frac{\mathrm{d}^{m} A_{n+1}^{(s+2)}}{\mathrm{d} \varepsilon^{m}}
\end{aligned}
$$

Mit Hülfe dieser Formeln und der in der Tafel enthaltenen Coefficienten kann man offenbar die $A_{n}^{(s)}$ für $s=3,5,7$ und $n<12$ leicht tabuliren. Der in dem ersten Gliede der rechten Seite der obigen Gleichungen auftretende Vergrösserungsfactor $\frac{s+2 m}{s}$ ist zwar nachtheilig, indessen wird dieser Nachtheil wieder dadurch aufgehoben, dass man die $A_{n}^{(s)}$ für höhere Werthe von $s$ nur mit geringerer Genauigkeit braucht als für niedere.

Beachtenswerth ist auch die einfache Gleichung:

$$
A_{n}^{(s+2)}=A_{n+1}^{(s+2)}-\frac{\mathrm{I}}{s} \frac{\mathrm{d} A_{n}^{(s)}}{\mathrm{d} \varepsilon}
$$

Die Tafel giebt die $A_{n}^{(1)}$ und $A_{12}{ }^{(3)}$ auf 7 Decimalen $A_{12}{ }^{(5)}$ auf $6, A_{12}{ }^{(7)}$ auf 5 Decimalen, so lange $\varepsilon-\varepsilon_{0}$ kleiner als 0.22 ist. Die letzte Decimale ist vielleicht in einigen Fällen nicht mehr ganz sicher, indessen ist die Genauigkeit der Tafel jedenfalls vöilig hinreichend. Da die Planeten vom Hilda.Typus alle nur wenig von einander verschiedene mittlere Bewegungen haben, so werden für dieselben in den meisten Fällen nur die allerersten Glieder der Reihen zu beachten sein. Ich habe jedoch der Tafel die vorliegende Ausdehnung gegeben, damit zwischen derselben und der Masal'schen 'Tafel keine Lücke bestehen bliebe, und damit sie auch für die bis jetzt entdeckten, dem Jupiter am nächsten kommenden Planeten anwendbar sei. Indessen ist zu bemerken, dass für (279) Thule die $A_{n}{ }^{(s)}$ aus der Tafel vielleicht nicht immer mit genügender Schärfe gefunden werden können. Doch ist für diesen Planeten die Grösse $\boldsymbol{A}=\varepsilon-\varepsilon_{0}$ positiv, die Reihen für die $A_{n}{ }^{(s)}$ wechseln also regelmässig das Zeichen, und man wird ohne Weiteres übersehen können, bis zu welcher Decimale die so gefundenen Werthe $\operatorname{der} A_{n}^{(s)}$ zuverlässig sind. Eine Erweiterung der Tafel in dieser Hinsicht wird übrigens nur wenig Mühe kosten.

Um die Richtigkeit der Coefficienten der Reihen zu prüfen, habe ich jede mechanische Quadratur doppelt ausgeführt, indem ich einmal die Werthe des Integranden für $\varphi=0^{\circ}, 10^{\circ}, 20^{\circ} \cdots 90^{\circ}$ und ein anderes Mal für $\varphi$ gleich $5^{\circ}, 15^{\circ} \cdots 85^{\circ}$ berechnete. Ferner habe ich den Umstand benutzt, dass die Tafel bis an die Masal'sche heranreicht und habe die fur $\log \alpha=9.84^{\circ}$ mit Hülfe der Tafel berechneten $A_{n}^{(s)}$ mit den aus der Masal'schen Tafel entnommenen Werthen verglichen.

Es folgt nunmehr die Tafel, Alle Zahlen, ausge. nommen das cunstante Glied einer jeden Reihe, sind Logarithmen, denen sämmtlich - 10 hinzuzusetzen ist. 


$$
2 \varepsilon=\frac{\alpha^{2}}{1-\alpha^{2}} \quad \Delta=\varepsilon-\varepsilon_{0} \quad \varepsilon_{0}=0.685 \mathrm{I} 038
$$

$$
\begin{aligned}
& A_{0}{ }^{(1)}=0.7966620-9.2695435 \Delta+8.961820 \Delta^{2}-8.738185 \Delta^{3}+8.5538 \Delta^{4}-8.392 \Delta^{5}+8.246 \Delta^{6}-8.11 \Delta^{2} \\
& A_{1}{ }^{(1)}=0.4408896-8.7932314 \Delta+8.357835 \Delta^{2}-8.04107 \Delta^{3}+7.7825 \Delta^{4}-7.558 \Delta^{5}+7.359 \Delta^{6} \\
& A_{2}{ }^{(1)}=0.3430054-8.5565288 \Delta+8.035713 \Delta^{2}-7.65260 \Delta^{3}+7.3390 \Delta^{4}-7.066 \Delta^{5}+6.81 \Delta^{6} \\
& A_{3}{ }^{(1)}=0.2914787-8.392711 \Delta+7.80554 \Delta^{2}-7.3693 \Delta^{3}+7.012 \Delta^{4}-6.70 \Delta^{5} \\
& A_{4}^{(1)}=0.2582027-8.265818 \Delta+7.62358 \Delta^{2}-7.1423 \Delta^{3}+6.746 \Delta^{4}-6.40 \Delta^{5} \\
& A_{5}^{(1)}=0.2343704-8.161633 \Delta+7.47198 \Delta^{2}-6.95^{14} \Delta^{3}+6.519 \Delta^{4}-6.15 \Delta^{5} \\
& A_{6}{ }^{(1)}=0.2161903-8.072965 \Delta+7.34 \mathrm{I} 44 \Delta^{2}-6.785^{6} \Delta^{3}+6.320 \Delta^{4} \\
& A_{7}^{(1)}=0.2017171-7.995^{635} \Delta+7.2265^{6} \Delta^{2}-663^{89} \Delta^{3}+6.149 \Delta^{4} \\
& A_{8}{ }^{(1)}=0.1898336-7.926981 \Delta+7.1237 \quad \Delta^{2}-6.507 \quad \Delta^{3}+5.99 \quad \Delta^{4} \\
& A_{9}{ }^{(1)}=0.1798453-7.865197 \Delta+7.0305 \quad \Delta^{2}-6.386 \quad \Delta^{3}+5.83 \quad \Delta^{4} \\
& A_{10}{ }^{(1)}=0.1712944-7.808997 \Delta+6.9453 \quad \Delta^{2}-6.277 \quad \Delta^{3}+5.73 \quad \Delta^{4} \\
& A_{11}{ }^{(1)}=0.163^{8646}-7.757431 \quad \Delta+6.8668 \quad \Delta^{2}-6.174 \quad \Delta^{3}+5.5^{8} \Delta^{4} \\
& A_{12}{ }^{(1)}=0.1573296-7.709774 \Delta+6.7939 \quad \Delta^{2}-6.079 \quad \Delta^{3}+5.47 \quad \Delta^{4} \\
& A_{12}{ }^{(3)}=0.1503059-8.135872 \Delta+7.41775 \Delta^{2}-6.8322 \Delta^{3}+6.294 \Delta^{4} \\
& A_{12}{ }^{(3)}=0.144061-8.309586 \Delta+7.71441 \Delta^{2}-7.2162 \Delta^{3}+6.779 \Delta^{4} \\
& A_{12}{ }^{(7)}=0.13^{847} \quad-8.41024 \quad \Delta+7.9016 \quad \Delta^{2}-7.4720 \quad \Delta^{3}+7.018 A^{4}
\end{aligned}
$$

Berlin 1896 März.

H. Ludendorff.

\section{Planet (316) Goberta.}

\section{Von A. Berberich.}

Aus den Beobachtungen 189 I Sept. 8, Oct. 5, Dec. I und 28 ergeben sich durch Variation der Distanzen des 1. und 4. Ortes die folgenden Elemente :

Epoche I 89 I Dec. 28.5 M. Z. Berlin.

$$
\begin{aligned}
& M=307^{\circ} 42^{\prime} 5^{\prime \prime} .5 \\
& \omega=307 \quad 552.0 \\
& \delta==\begin{array}{lll}
24 & 34 & 27.0
\end{array} \quad 1900.0 \\
& i=2 \mathrm{I} 837.8 \\
& \varphi=8^{2} \quad 5^{20.3} \\
& \mu=625.609 \\
& \log a=0.502469
\end{aligned}
$$

Mittlere Oerter.

$$
\begin{aligned}
& 1891 \text { Oct. } 5 \mathrm{~d} \lambda=-0 . \mathrm{r} \quad \mathrm{d} \beta=-0.2 \\
& \text { Dec. } 1=+0.1=+3.8
\end{aligned}
$$

Für die recht günstige II. Opposition im December 1892 verschiebt sich der Planetenort nach den neuen Elementen um ca. $+3^{m} \cdot 8+4^{\circ}$ gegen die damals an die Herren Charlois und Wolf mitgetheilte Ephemeride. Herr Wolf, der auch jetzt auf seinen damals gemachten Aufnahmen den Planeten nicht finden kann, ist der Ansicht, dass die Helligkeit geringer war als sie berechnet ist (12.7 Gr.).

In Folge der Jupiterstörungen haben sich übrigens

Berlin, Kgl. Recheninstitut, Neujahr 1896 . die Elemente nach 1891 nicht unbeträchtlich verändert. In Conjunction mit dem Jupiter stand Planet (3 I6) am 1. Sept. 1891 bei einer gegenseitigen Entfernung $\boldsymbol{\Delta}=1.87$.

Die Störungen, berechnet in 20 tägigen Intervallen vom 8. Sept. 1891 bis 31 . Dec. 1892 betragen:

$$
\begin{aligned}
& \Delta M=-16^{\prime} \quad 3.63 \\
& \Delta \omega=+22 \quad 18.62 \\
& \Delta \delta=-213.01 \\
& \Delta i=-0 \quad 1.63 \\
& \Delta \varphi=-721.73 \\
& \Delta \mu=+2 \text {. 1 } 2923
\end{aligned}
$$

Als Elemente, mit denen man in Zukunft die Oerter des Planeten Goberta zu rechnen hätte, kann man hiernach einstweilen folgende nehmen:

Epoche 1893 Jan. 0.0 M. Z. Berlin.

$$
\begin{aligned}
& M=11^{\circ} 29^{\prime} \quad 4^{\prime \prime} 9 \\
& \omega=30728 \quad 10.6 \\
& 8=\begin{array}{lll}
24 & 32 & 14.0
\end{array} \quad 1900.0 \\
& i=2183^{6.5} \\
& \varphi=75758.6 \\
& \mu=627.7382 \\
& \log a=0.501585
\end{aligned}
$$

